\title{
Análisis comparativo in vitro de la resistencia adhesiva al cizallamiento de brackets metálicos adheridos a superficies dentarias tratadas con diferentes agentes blanqueadores.
}

\section{Comparative in vitro analysis of the adhesive resistance to the shear bond strength of metal brackets adhered to dental surfaces treated with different bleaching agents.}

\author{
Victor Rojas ${ }^{1 *}$, Manuel Ignacio Gómez ${ }^{1}$, Camila Sampaio², Mackarena Sáez ${ }^{3}$, Rodrigo Oyonarte ${ }^{1}$
}

\author{
1. Departamento de Ortodoncia, Facultad de \\ Odontología Universidad de los Andes. Santiago, \\ Chile. \\ 2. Departamento Odontología Restauradora, \\ Facultad de Odontología Universidad de los Andes. \\ Santiago, Chile. \\ 3. Práctica privada, Santiago, Chile. \\ * Correspondencia Autor: Victor Rojas | Dirección: \\ Monseñor Álvaro del Portillo 12.455, Las Condes, \\ Santiago, Chile. | Teléfono: +5699479 7710| \\ E-Mail:vhrojas@miuandes.cl \\ Trabajo recibido el 18/05/2020. \\ Trabajo revisado 06/07/2020 \\ Aprobado para su publicación el 23/08/2020
}

\begin{abstract}
RESUMEN
Objetivo: Evaluar y comparar el efecto de tres agentes blanqueadores en la resistencia adhesiva al cizallamiento de brackets metálicos. Método: Se cementaron 76 brackets en premolares humanos extraídos por indicación de ortodoncia. Se establecieron 4 grupos: (0) control, (1) esmalte tratado con Peróxido de Carbamida al 16\%, (2) Peróxido de Hidrógeno al $35 \%$ y (3) tiras de blanqueamiento con Peróxido de Hidrógeno al $10 \%$. Los brackets fueron cementados con Transbond XT (3M Unitek, EE.UU.). Se realizaron 5.000 ciclos de termociclado, luego se evaluó la resistencia adhesiva mediante una máquina Bisco y la cantidad de material adhesivo remanente según el índice adhesivo remanente (ARI). El análisis estadístico incluyó pruebas de Shapiro- Wilk, Kruskal -Wallis y Mann-Whitney. Resultados: Los grupos $0,1,2,3$ obtuvieron fuerzas de resistencia de $12.64,11.06,6.4$ y 9.34 Mpa respectivamente con diferencia estadística entre los grupos $(p<0.05)$. EI ARI fue significativamente diferente en algunos grupos $(p<0.05)$, indicando fallas adhesivas para el grupo $2(p<0.05)$, cohesivas para el grupo control y de ambos tipos para los grupos 1 y 3 . Conclusiones: El peróxido de hidrógeno al $35 \%$ reduce la resistencia adhesiva al cizallamiento en brackets metálicos sometidos a termociclado 24 horas luego del blanqueamiento.
\end{abstract}

PALABRAS CLAVE

Bracket ortodoncia; Fuerza de cizalla; Blanqueamiento dental.

Int. J. Inter. Dent Vol. 14(1); 17-21, 2021.

\section{ABSTRACT}

Aim: To evaluate and compare the effect of three bleaching agents on the shear bond strength of metallic brackets. Method: Brackets were cemented on 76 human premolars extracted for orthodontic reasons. 4 groups were evaluated: (0) Control, (1) enamel treated with Carbamide Peroxide 16\%, (2) Hydrogen Peroxide $35 \%$ and (3) Hydrogen Peroxide $10 \%$ "Whitening strips". All brackets were cemented using Transbond XT adhesive system (3M, Unitek, USA). All samples were thermocycled (5.000 cycles). Shear bond strength was evaluated with a Bisco machine and the amount of adhesive material at the base of the brackets and enamel surface was assessed using the adhesive remnant index (ARI). The statistical analysis included Shapiro-Wilk, KruskalWallis and Mann-Whitney $U$ tests. Results: The results of shear bond strength for groups $0,1,2$, and 3 were 12.64, 11.06, 6.4 and 9.34 MPa respectively, with statistically significant differences between groups $(p<0.05)$. ARI index was significantly different in some groups $(p<0.05)$, indicating cohesive failure for control, adhesive failure for group $2(p<0.05)$, adhesive and cohesive failure for groups 1 and 3 . Conclusions: The $35 \%$ hydrogen peroxide office bleaching agent reduces adhesion values in the metal brackets thermocycled 24 hours after bleaching.

KEY WORDS

Orthodontic bracket; Shear bond strength; Dental bleaching.

Int. J. Inter. Dent Vol. 14(1); 17-21, 2021. 


\section{INTRODUCCIÓN}

La ortodoncia y el blanqueamiento dental son dos procedimientos a los cuales los pacientes recurren frecuentemente para optimizar la estética de la sonrisa. Se han introducido distintos métodos de blanqueamientos como el uso del Peróxido de Hidrógeno, Peróxido de Carbamida y Perborato de Sodio(1). Comúnmente, estos se clasifican de acuerdo al lugar en donde se realizan, ya sea en la oficina o en la casa $^{(2)}$.

El tratamiento en oficina se realiza con altas concentraciones de Peróxido de Hidrógeno (25-35\%), el cual corresponde a un líquido capaz de oxidar compuestos orgánicos e inorgánicos causando decoloración ${ }^{(3)}$. Por otro lado, para el tratamiento en casa existen distintas presentaciones, conteniendo en su mayoría Peróxido de Carbamida en variadas concentraciones, siendo la más común al $10 \%{ }^{(4)}$.

Se han estudiado los cambios estructurales que provocaría en el esmalte dentario los distintos tipos de agentes blanqueadores, como por ejemplo, aumento de la porosidad del esmalte, pérdida de contenido mineral y alteración de su estructura prismática. Sin embargo, aún es controversial sus efectos en la adhesión de materiales dentales, y específicamente en relación a la resistencia adhesiva en ortodoncia ${ }^{(1}$ $5,6)$. Hay autores ${ }^{(6,7)}$ que reportan que no existen diferencias en las resistencias adhesivas de los brackets de ortodoncia cuando los dientes son blanqueados previamente, sin embargo existen numerosos autores que reportan estas diferencias ${ }^{(1,5,8)}$

Las caracteristicas y cualidades de la adhesión del bracket y el diente dependen de la interacción entre dos interfases: interfase resina-bracket y la interfase resina-esmalte dentario. Se considera clínicamente aceptable si los valores de resistencia a las fuerzas de cizalla son de 6 a $8 \mathrm{Mpa}$. Valores mayores a $13 \mathrm{MPa}$ pueden aumentar el riesgo de fractura de esmalte ${ }^{(9)}$

Se ha sugerido que los cambios registrados en la forma del esmalte, cuando se utilizan agentes blanqueadores, serían reversibles y a los 3 meses ya no habrían signos de alteración morfológica(10,11).

Debido a que existen distintos protocolos de blanqueamiento y a su alta demanda por parte de los pacientes, es de gran interés estudiar cómo puede verse afectada la adhesión de cementos ortodóncicos de resina compuesta al esmalte con estos tratamientos. El presente estudio in vitro tiene como propósito comparar la resistencia adhesiva a las fuerzas de cizallamiento de los brackets metálicos cementados en dientes humanos, 24 horas después de ser sometidos a diferentes tipos de blanqueamiento dental y un proceso de termociclado, en relación a dientes con superficies dentarias no tratadas como control.

\section{MATERIAL Y MÉTODO}

Se realizó un estudio experimental in vitro, utilizando premolares humanos extraídos por indicación de ortodoncia (adultos y niños). Una vez extraídos fueron almacenados en agua potable (de la llave). El estudio fue analizado y aprobado por el comité de ética científica de la Universidad de los Andes, Chile.

Previo a la extracción, se solicitó la autorización y consentimiento informado por parte del paciente y/o apoderado (en caso de paciente menor de edad) para la donación de los dientes con fines científicos. Los criterios de selección de los dientes incluyeron: Indicación de extracción por ortodoncia, dientes sanos (sin caries ni restauraciones), con anatomía normal y sin daños en el esmalte debido al proceso de extracción. Se excluyeron todos aquellos dientes con restauraciones previas, con caries, con algún tratamiento de superficie previo y que no presentaban anatomía normal ó presentaban daños.

El tamaño de la muestra para un diseño factorial fue calculado con el programa $\mathrm{G}^{*}$ Power 3.1 considerando un tamaño del efecto medio $(f=0,4)$, una potencia del $80 \%$ y un nivel alfa del $5 \%$. Este análisis determinó un tamaño total de muestra de 76 dientes divididos en 4 grupos de 19 dientes cada uno.

Tres grupos recibieron distintos tratamientos blanqueadores y un control sin tratamiento. En todos los premolares se cementaron brackets nuevos (Gemini, slot 0.022, 3M Unitek, USA). Se realizó grabado ácido con ácido ortofosfórico al $37 \%$ y luego el protocolo adhesivo descrito por el fabricante utilizando el sistema de composite de fotocurado Transbond XT (3M, Unitek, USA) en el centro de las caras libres de los premolares (vestibular o lingual/palatina), siguiendo el eje longitudinal de la corona. La cementación de todos los brackets fue realizada por el mismo operador calibrado.

En el grupo 0 (control) los premolares no recibieron ningún tratamiento blanqueador. En el grupo 1 los premolares fueron tratados con Peróxido de Carbamida 16\% (BM4-Brasil Material and Instrumentals Ltda). Se realizó el protocolo descrito por el fabricante, aplicando 2 veces al día en la superficie dentaria por 30 minutos cada aplicación, durante
2 días consecutivos, repitiendo el procedimiento al día siguiente. El grupo 2 fue tratado con Peróxido de Hidrógeno 35\% (Pola Office SDI). Se aplicó una delgada capa, 2 aplicaciones al día durante 8 minutos cada una aplicando luz Led, durante 2 días consecutivos (el fabricante recomienda 3 o 4 aplicaciones máximo). Por último, en el grupo 3, los premolares fueron tratados con Peróxido de Hidrógeno 10\% Whitening strip Oral B. Se aplico 2 veces al día en la superficie dentaria y se dejaba actuar 30 minutos cada aplicación, en 2 días consecutivos. Las muestras fueron conservadas en agua potable (de la llave) entre cada ciclo de blanqueamiento.

En cada grupo experimental se cementaron brackets metálicos 24 horas despúes de realizar el blanqueamiento. Se sometieron a termociclado de 5.000 ciclos de $5^{\circ} \mathrm{C}$ y $55^{\circ} \mathrm{C}$ simulando un envejecimiento natural de 6 a 8 meses. Las muestras se dispusieron en bloques de acrílico de autocurado dejando expuesta la superficie de la corona con el bracket. Posteriormente fueron desalojados mediante fuerzas de cizalla utilizando una máquina Bisco (Shear Bond Tester ${ }^{\circledR}$, Bisco Dental, Schaumburg, Illinois, EE. UU) (Figura 1), registrando su magnitud en Megapascales (MPa).

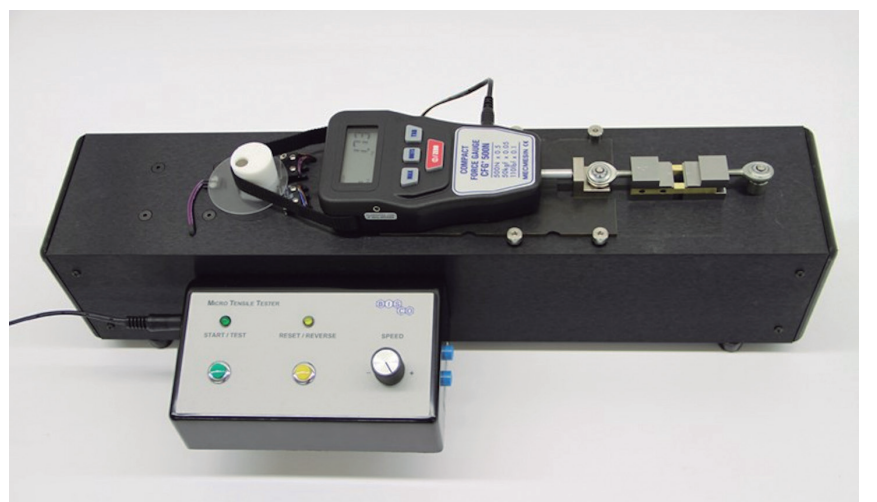

Figura 1. Máquina Bisco para registro de fuerza de cizalla

Los cambios en la base del bracket y superficie del esmalte fueron examinados cualitativamente bajo un microscopio óptico (Microscopio óptico Leica DMLP con lente $11,2 \mathrm{~mm}$ de diámetro y magnificación $2,5 x$ ) por un solo operador calibrado y ciego (Figura 2). Se estableció el índice adhesivo remanente (ARI) según Artun y Bergland (1984) para determinar las fallas adhesivas o cohesivas ${ }^{(12)}$. En la tabla 1 podemos encontrar la descripción de éste índice.

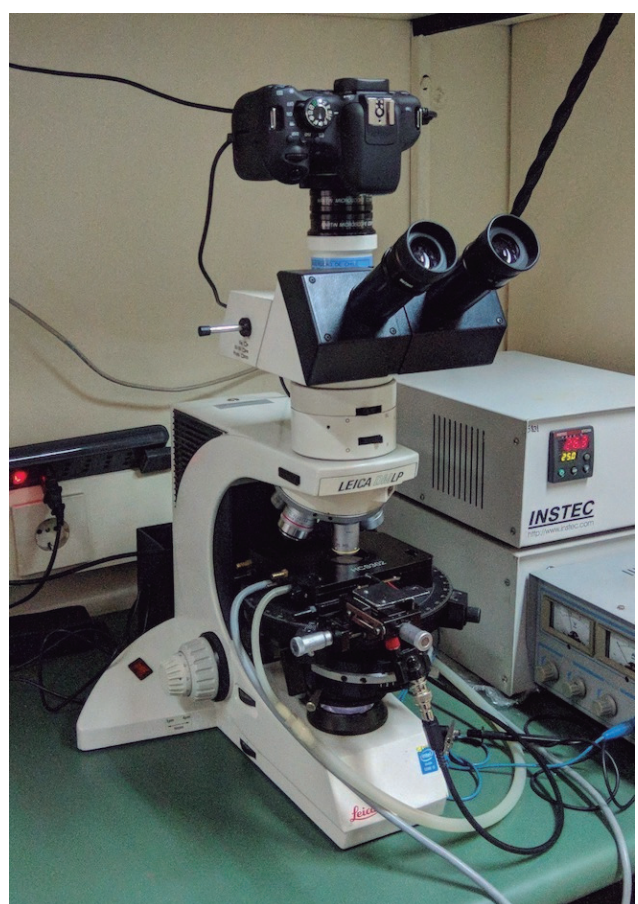

Figura 2. Microscopio óptico utilizado para evaluar ARI 
Tabla 1: Descripción índice de Remanente Adhesivo (ARI)

\begin{tabular}{|c|c|c|}
\hline ARI & Definición & Tipo de falla \\
\hline 0 & $\begin{array}{l}\text { No hay remanente adhesivo } \\
\text { en la superficie dental }\end{array}$ & \multirow{2}{*}{$\begin{array}{c}\text { Falla en la interfase entre } \\
\text { dos estructuras, falla tipo } \\
\text { adhesiva }\end{array}$} \\
\hline 1 & $\begin{array}{l}\text { Menos de la mitad del } \\
\text { remanente adhesivo en la } \\
\text { estructura dental }\end{array}$ & \\
\hline 2 & $\begin{array}{c}\text { Más de la mitad del } \\
\text { remanente adhesivo en la } \\
\text { estructura dental }\end{array}$ & \multirow{2}{*}{$\begin{array}{c}\text { Falla al interior de la } \\
\text { estructura del material, falla } \\
\text { tipo cohesiva }\end{array}$} \\
\hline 3 & $\begin{array}{l}\text { Todo el resto de remanente } \\
\text { adhesivo permanece en la } \\
\text { estructura dental }\end{array}$ & \\
\hline
\end{tabular}

\section{Análisis estadístico}

La distribución muestral en cada grupo se analizó utilizando el test de Shapiro-Wilk, determinándose que los datos de la muestra no distribuían en forma normal. En consecuencia, el test no paramétrico Kruskal-Wallis fue utilizando para la comparación entre grupos y el test de Mann-Whitney para la comparación entre pares de grupos, tanto para data sobre resistencia adhesiva al cizallamiento como para la evaluación de valores de ARI. La significación estadística fue predeterminada a un $95 \%$ de confianza (valor $p \leq 0.05$ ).

\section{RESULTADOS}

Los grupos fueron evaluados para normalidad por el test de ShapiroWilk, en donde la variable resistencia adhesiva al cizallamiento fue no normal en el grupo control $(p=0,242)$.

El análisis estadístico de la resistencia adhesiva al cizallamiento en los diferentes grupos es representado en gráfico de caja en la figura 3 . Los resultados muestran que todos los grupos experimentales obtuvieron menor resistencia adhesiva a las fuerzas de cizallamiento que el grupo control, el cual obtuvo una mediana de $12,64 \mathrm{MPa}$ de resistencia (7,92 MPa- 20,92 MPa), seguido por el grupo de Peróxido de Carbamida $16 \%$ con $11,06 \mathrm{MPa}(3,79 \mathrm{MPa}-24,46 \mathrm{MPa})$, grupo de Peróxido de hidrógeno $10 \%$ con $9,34 \mathrm{MPa}(2,17 \mathrm{MPa}-23,13 \mathrm{MPa})$ y el grupo de Peróxido de Hidrógeno $35 \%$ con $6,4 \mathrm{MPa}(0,91 \mathrm{MPa}-17,72$ $\mathrm{MPa}$ ). Todos los grupos experimentables presentaron muestras cuya resistencia adhesiva fue inferior al límite aceptable, en el grupo 1 un $15,78 \%$ de las muestras tuvo ese resultado, en el grupo 2 un $57,89 \%$ y en el grupo 3 un $31,57 \%$.

Comparación de la resistencia a las fuerzas de cizalla.

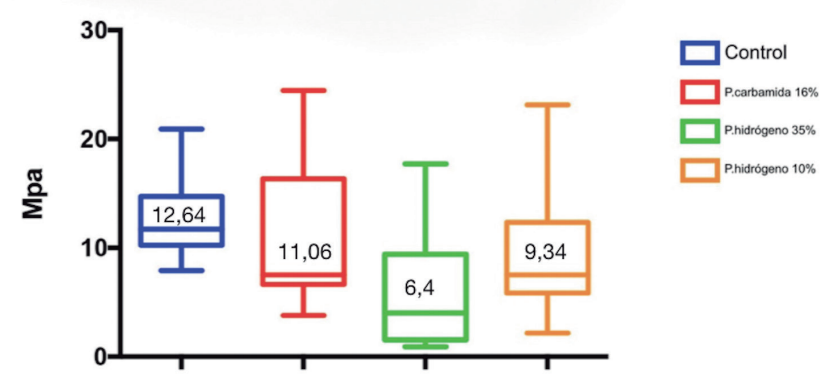

Grupos

Fuerza de resistencia adhesiva al cizallamiento (MPa). Grupo 0: Control, Grupo 1: Peróxido de Carbamida $16 \%$, Grupo 2: Peróxido de Hidrógeno $35 \%$, Grupo 3 : Whitening Strips.

Figura 3. Comparación de la resistencia a las fuerzas de cizalla entre los grupos evaluados

La prueba de Kruskal-Wallis mostró diferencias estadísticamente significativas (DES) entre los grupos ( $\leq \leq 0,05)$, mientras que la evaluación de pares de grupos con la prueba de Mann-Whitney mostró diferencias estadísticamente significativas donde el grupo control fue mayor que el grupo Peróxido de Hidrógeno al 35\% ( $p=0,001)$, grupo control fue mayor que el grupo Peróxido de Hidrógeno al $10 \%$ Whitening strips $(p=0,014)$ y grupo Peróxido de Carbamida $16 \%$ fue mayor que
Tabla 2: Índice de Remanente Adhesivo (ARI) de los grupos estudiados

\begin{tabular}{c|c|c|c|c|c}
\hline \multicolumn{6}{c}{ Grupos } \\
\hline ARI & Control & $\begin{array}{c}\text { Peróxido } \\
\text { Carbamida } \\
16 \%\end{array}$ & $\begin{array}{c}\text { Peróxido de } \\
\text { Hidrógeno } \\
35 \%\end{array}$ & $\begin{array}{c}\text { Whitening } \\
\text { strips }\end{array}$ & Total \\
\hline 0 & 5 & 4 & 16 & 6 & 31 \\
\hline 1 & 2 & 9 & 2 & 5 & 18 \\
\hline 2 & 12 & 5 & 1 & 6 & 24 \\
\hline 3 & 0 & 1 & 0 & 2 & 3 \\
\hline Total & 19 & 19 & 19 & 19 & 76 \\
\hline
\end{tabular}

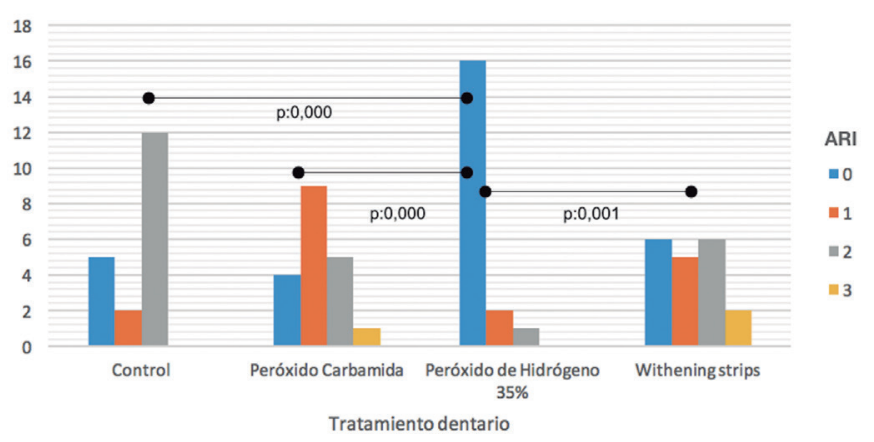

Frecuencia del valor del Índice de Adhesivo Remanente de los 4 grupos evaluados y testeados con la prueba de Mann- Whitney.

Las comparaciones de pares fueron estadísticamente significativas para 3 pares de grupos, Grupo control y Peróxido de Hidrógeno al $35 \%$, Grupo Peróxido de carbamida $16 \%$ y Peróxido de Hidrógeno al $35 \%$ y grupo Peróxido de Hidrógeno al $35 \%$ y Peróxido de Hidrógeno al $10 \%$ Whitening Strips. $(p=0,000, p=0,000, p=0,001$ respectivamente)

Figura 4. Gráfico de comparación entre pares para los resultados de Índice Remanente Adhesivo (ARI)

grupo peróxido de Hidrógeno al $35 \%(p=0,030)$ Los otros pares no mostraron diferencias estadísticamente significativas.

Por otro lado, el análisis descriptivo y estadístico del Índice ARI se presentan en la tabla 2 y figura 4 . En todos los grupos experimentales hubo muestras que presentaron fallas adhesivas o cohesivas. En el grupo control hubo mayor frecuencia de puntuación ARI 2 que nos indica que hubo, en su mayoría, fallas cohesivas dentro de la resina. En el grupo 1 (Peróxido de Carbamida 16\%) y en el grupo 3 (Whitening Strips, Peróxido de Hidrógeno 10\%) se identificaron fallas adhesivas mayormente (índices 0 y 1 ), aunque de igual forma hubo muestras que presentaron índices 2 y 3 indicando fallas cohesivas. Para el grupo 2 (Peróxido de Hidrógeno 35\%) el índice fue principalmente 0 indicando una falla adhesiva en la mayoría de las muestras.

Se encontraron diferencias significativas $(p=0.000)$ entre los grupos con la prueba de Kruskal Wallis, lo que muestra que al menos un grupo fue estadísticamente diferente de los otros. La prueba de MannWhitney mostró diferencias significativas entre 3 pares de grupos. El grupo control obtuvo mayor índice que el grupo Peróxido de Hidrogeno al $35 \%(p=0,000)$, grupo Peróxido de Hidrógeno obtuvo menor índice que el grupo Peróxido de Carbamida $16 \%(p=0,000)$ y grupo Peróxido de Hidrógeno al $35 \%$ obtuvo menor índice que el grupo Peróxido de Hidrógeno 10\% (Whitening Strips) $(p=0,001)$.

\section{DISCUSIÓN}

Son diversos los estudios que reportan que la realización de tratamientos de blanqueamiento dental, independiente de los agentes blanqueadores utilizados, reducirian la resistencia adhesiva a las fuerzas de cizallamiento en diferentes grados ${ }^{(5,7,13,14)}$. Esto es consistente con los resultados reportados en el presente estudio.

Reynolds et al. sugieren que una fuerza de unión mínima de 6 a 8 Mpa es adecuada para la mayoría de los casos clínicos de ortodoncia, y que valores mayores a $13 \mathrm{Mpa}$ se pueden asociar a posible fractura del esmalte ${ }^{(9)}$. En nuestro estudio todas las muestras del grupo control 
obtuvieron valores mayores a la mínima aceptable. Sin embargo, en los grupos experimentales, hubo numerosas muestras que presentaron valores de resistencia inferior a esta mínima. Esto ocurrió especialmente el grupo tratado con Peróxido de Hidrógeno al 35\%, el cual presentó un $57,89 \%$ de sus muestras con resultados inferiores al límite aceptable y cuya mediana se encontró muy cercana a este. Ello implica que la fuerza adhesiva lograda no es consistentemente superior a los mínimos aceptables en todos los grupos experimentales.

Respecto a lo anterior, se han propuesto distintos tiempos de cementación de brackets posterior al blanqueamiento, con el fin de mejorar la adhesión. Algunos estudios reportan que, como mínimo, debería retrasarse 2 semanas la cementación de materiales dentales después de un blanqueamiento(15, 16,17, 18). Los hallazgos de Bishara et al. no suponen diferencia significativa entre la fuerza de unión al cizallamiento de dientes blanqueados y no blanqueados si la unión se realiza 2 semanas después del blanqueamiento ${ }^{(6)}$.Josey et al. concluyen que el Peróxido de Hidrógeno residual se disuelve entre 1 y 6 semanas, por lo que la resistencia al cizallamiento aumentó al cementar los brackets después de 6 semanas del blanqueamiento(10). Por otra parte, Scougall et al. concluyeron que el uso de peróxidos antes de la cementación de brackets debería estar contraindicada, o retrasarse como mínimo 7 días e idealmente 30 días, para mejorar los valores de adhesión en dientes blanqueados ${ }^{(19)}$.

Nuestros resultados concuerdan con los presentados en los estudios anteriores, en cuanto a la disminución de la resistencia adhesiva al cizallamiento de brackets cementados 24 horas después de tratar con agentes blanqueadores, especialmente en el grupo de Peróxido de Hidrógeno al $35 \%$. Es importante destacar que aún cuando se haya realizado el proceso de termociclado, simulando un envejecimiento natural de nuestras muestras, los resultados promedios de resistencia fueron consistentemente menores al grupo control, al cementar los brackets 24 horas después del blanqueamiento dental.

El índice ARI se utiliza para definir el sitio de falla de unión entre el esmalte, el adhesivo y la superficie de unión, que en este caso son los brackets ${ }^{(8)}$. Los resultados de este parámetro mostraron diferencias entre los cuatro grupos evaluados. Estas variaron desde la presencia de fallas cohesivas en el grupo control, progresando a fallas adhesivas en los grupos experimentales, especialmente con el Peróxido de Hidrógeno al $35 \%$. Esta tendencia coincide con los resultados obtenidos por Patusco et al. en el grupo tratado con peróxido de hidrógeno al $35 \%$ donde el ARI tiende a $0^{(5)}$.

Se ha propuesto que el índice ARI tiende a ser más elevado al obtener mayores valores de resistencia adhesiva a las fuerzas de cizallamiento, al igual como sucedió con nuestro grupo control, el cual tuvo en su mayoría índice ARI 2 indicando falla cohesiva(19, 20). Scougall et al. proponen que queda menos cantidad de adhesivo en la superficie dental al realizar blanqueamiento con peróxido, ya que los agentes químicos pueden causar una falla en la interfase adhesiva del esmalte ${ }^{(19)}$. Esto explicaría nuestros resultados en los grupos experimentales (grupos 1 , 2 y 3), los cuales en su mayoría presentaron fallas de tipo adhesiva, presentando nada o menos de la mitad de adhesivo remanente sobre la superficie del esmalte en casi todas las muestras.

Existen diferencias metodológicas respecto de otros estudios sobre el tema, lo cual no permite una comparación directa de todos los resultados. Dentro de los factores que robustecen el presente estudio podemos mencionar el uso del termociclado, que permite acercarnos a las condiciones de la cavidad oral, dado que los adhesivos de ortodoncia están sujetos diariamente entre 20 y 50 ciclos de cambio de temperatura(21). Se optó por someter las muestras a 5.000 ciclos, a fin de simular las condiciones de permanencia del adhesivo de ortodoncia en boca, esto se asemeja más a la realidad bucal respecto de otros protocolos de aproximadamente 1.000 ciclos $^{(22,23)}$. Otro factor importante es la utilización de dientes humanos, al comparar los resultados con otros estudios hechos en dientes de bovinos ${ }^{(16,17,19,20,24)}$

Nuestros resultados indican un deterioro inmediato en la capacidad adhesiva de los brackets ortodóncicos cementados con resina al esmalte dentario, secundaria a la utilización de agentes blanqueadores, el que es persistente y tendría un efecto a largo plazo. Dado lo anterior, no es aconsejable realizar la cementación de aparatos ortodóncicos con cementos de resina 24 horas después de realizado un blanqueamiento dental.

\section{CONCLUSIONES}

- El blanqueamiento afecta significativamente la resistencia de fuerza adhesiva al cizallamiento de los brackets en el esmalte humano.

- El uso de Peróxido de Hidrógeno 35\% reduce significativamente los valores de resistencia adhesiva al cizallamiento.

- Es aconsejable retrasar el tratamiento de con agentes blanqueadores antes de iniciar un tratamiento de ortodoncia, sobre todo si se utilizara Peróxido de Hidrógeno al 35\%.

- El índice ARI es mayor cuanto mayor es la resistencia adhesiva a las fuerzas de cizallamiento.

\section{RELEVANCIA CLÍNICA}

El blanqueamiento dental es un procedimiento a los cuales los pacientes recurren para mejorar la estética de la sonrisa. Debido a esto, se justifica científicamente la realización de un estudio para evaluar los efectos del blanqueamiento dental en relación con la resistencia adhesiva de los brackets en ortodoncia. Los resultados principales del estudio indican que el uso de agentes blanqueadores, y sobre todo el peróxido de hidrogeno al $35 \%$, afecta la resistencia de los brackets a las fuerzas de cizallamiento, por lo cual se sugiere como consecuencia práctica, postergar su uso antes de iniciar un tratamiento de ortodoncia.

\section{AGRADECIMIENTO}

A la empresa 3M Unitek por la donación de los brackets y cementos utilizados en el estudio.

\section{FUENTE DE FINANCIAMIENTO Y CONFLICTO DE INTERÉS}

Este trabajo no cuenta con financiamiento alguno. Los autores de este estudio declaran no presentar ningún tipo de conflicto de interés. Ninguna compañía u otra entidad recibió beneficio directo o indirecto proveniente de los resultados de este trabajo.

\section{Bibliografía}

1. Vahid-Dastjerdi E, Khaloo N, Mojahedi SM, Azarsina M. Shear bond strength of orthodontic brackets to tooth enamel after treatment with different tooth bleaching methods. Iran Red Crescent Med J. [edición electronica]. 2015. [citado 17 mayo 2020]; Disponible en: https://www.ncbi.nlm.nih.gov/pmc/articles/PMC4698138/ 2. Berga-Caballero A, Forner-Navarro L, Amengual-Lorenzo J. At-home vital bleaching: a comparison of hydrogen peroxide and carbamide peroxide treatments. Med Oral Patol Oral Cir Bucal. 2006;11:94-9

3. Joiner A. The bleaching of teeth: A review of the literatura. J Dent. 2006; 34(7):412-9

4. Bertone N, Zaiden S. Blanqueamiento dentario. Aplicaciones clínicas. Revista de la Facultad de Odontología (UBA). 2008;23:19-25.

5. Patusco VC, Montenegro G, Lenza MA, Alves de Carvalho A. Bond strength of metallic brackets after dental bleaching. Angle Orthod. 2009;79(1):122-6.

6. Bishara SE, Oonsombat C, Soliman MM, Ajlouni R, Laffoon JF. The effect of tooth bleaching on the shear bond strength of orthodontic brackets. Am J Orthod Dentofacial Orthop. 2005; 128:755-60.

7. Uysal T, Basciftci FA, Usumez S, Sari Z, Buyukerkmen A. Can previously bleached teeth be bonded safely?. Am J Orthod Dentofacial Orthop. 2003;123:62832.

8. Gungor AY, Ozcan E, Alkis H, Turkkahraman H. Effects of different bleaching methods on shear bond strengths of orthodontic brackets. Angle Orthod. 2013;83(4):686-90
9. Reynolds IR. A review of direct orthodontic bonding. Br J Orthod. 1975;2(3):1718.

10. Josey AL, Meyers IA, Romaniuk K, Symons AL. The effect of a vital bleaching technique on enamel surface morphology and the bonding of composite resin to enamel. J Oral Rehabil. 1996;23:244-50.

11. Turkun M, Sevgican F, Pehlivan $Y$, Aktener BO. Effects of $10 \%$ carbamide peroxide on the enamel surface morphology: a scanning electron microscopy study. J Esthet Restor Dent. 2002;14:238-44.

12. Artun J, Bergland S. Clinical trials with crystal growth conditioning as an alternative to acid-etch enamel pretreatment. Am J Orthod. 1984;85(4):333-40.

13. Miles PG, Pontier JP, Bahiraei D, Close J. The effect of carbamide peroxide bleach on the tensile bond strength of ceramic brackets: an in vitro study. Am J Orthod Dentofacial Orthop. 1994:106:371-5.

14. Cacciafesta V, Sfondrini MF, Stifanelli P, Scribante A, Klersy C. The effect of bleaching on shear bond strength of brackets bonded with a resin-modified glass ionomer. Am J Orthod Dentofacial Orthop. 2006;130:83-7.

15. Cavalli V, Reis AF, Giannini M, Ambrosano GM. The effect of elapsed time following bleaching on enamel bond strength of resin composite. Oper Dent. 2001;26:597-602.

16. Spyrides GM, Perdigao J, Pagani C, Araujo MA, Spyrides SM. Effect of whitening agents on dentin bonding. J Esthet Dent. 2000;12:264-70.

17. De Almeida A, Lima D, Pereira A, Sousa S, Alves C. Influence of delay between 
dental bleaching with $35 \%$ hydrogen peroxide and orthodontic brackets on the bond strength at the enamel/adhesive interface. J Clin Exp Dent. 2019;11(5):44751 .

18. Nascimento GCR, de Miranda CA, Machado SMM, Brandão GAM, de Almeida $\mathrm{HA}$, Silva CM. Does the time interval after bleaching influence the adhesion of orthodontic brackets?. Korean J Orthod. 2013;43(5):242-7.

19. Scougall-Vilchis RJ, Gonzalez-Lopez BS, Contreras-Bulnes R, RodriguezVilchis LE, Garcia-Niño de Rivera MW, Kubodera-Ito T. Influence of four systems for dental bleaching on the bond strength of orthodontic brackets. Angle Orthod. 2011;81(4):700-6.

20. Pithon MM, Dos Santos RL, De Oliveira MV, Ruellas AC, Romano FL. Metallic brackets bonded with resin-reinforced glass ionomer cements under different enamel conditions. Angle Orthod. 2006;76(4):700-4.
21. Gale MS, Darvell BW. Thermal cycling procedures for laboratory testing of dental materials restorations. J Dent. 1999;27:89-99.

22. Sung EC, Chan SM, Mito R, Caputo AA. Effect of carbamide peroxide bleaching on the shear bond strength of composite to dental bonding agent enhanced enamel. J Prosthet Dent. 1999;82(5):595-9.

23. Khosravanifard $B$, Rakhshan $\mathrm{V}$, Araghi S, Parhiz $\mathrm{H}$. Effect of ascorbic acid on shear bond strength of orthodontic brackets bonded with resin-modified glass-ionomer cement to bleached teeth. J Dent Res Dent Clin Dent Prospects. 2012;6(2):59-64.

24. Titley KC, Torneck CD, Smith DC, Chernecky R, Adibfar A. Scanning electron microscopy observations on the penetration and structure of resin tags in bleached and unbleached bovine enamel. J Endod. 1991;17(2): 72-5. 